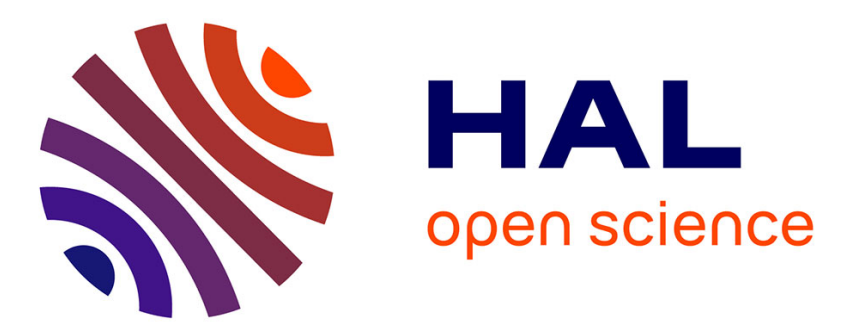

\title{
Least Restrictive and Minimally Deviating Supervisor for Safe Semi-Autonomous Driving at an Intersection: An MIQP Approach
}

Florent Altché, Xiangjun Qian, Arnaud de La Fortelle

\section{- To cite this version:}

Florent Altché, Xiangjun Qian, Arnaud de La Fortelle. Least Restrictive and Minimally Deviating Supervisor for Safe Semi-Autonomous Driving at an Intersection: An MIQP Approach. 2016 IEEE 19th International Conference on Intelligent Transportation Systems (ITSC 2016), Nov 2016, Rio de Janeiro, Brazil. hal-01360137

HAL Id: hal-01360137

https://hal-mines-paristech.archives-ouvertes.fr/hal-01360137

Submitted on 6 Sep 2016

HAL is a multi-disciplinary open access archive for the deposit and dissemination of scientific research documents, whether they are published or not. The documents may come from teaching and research institutions in France or abroad, or from public or private research centers.
L'archive ouverte pluridisciplinaire HAL, est destinée au dépôt et à la diffusion de documents scientifiques de niveau recherche, publiés ou non, émanant des établissements d'enseignement et de recherche français ou étrangers, des laboratoires publics ou privés. 


\title{
Least Restrictive and Minimally Deviating Supervisor for Safe Semi-Autonomous Driving at an Intersection: An MIQP Approach
}

\author{
Florent Altché ${ }^{2,1}$, Xiangjun Qian ${ }^{1}$ and Arnaud de La Fortelle ${ }^{1}$
}

\begin{abstract}
Although significant progress has been made in the last few years towards cooperative and autonomous driving, the transition from human-driven to fully automated vehicles is expected to happen slowly. The question of semi-autonomous driving, where Advanced Driver Assistance Systems assist human drivers in their decisions, will therefore become increasingly important. In this paper, we consider the problem of safe intersection crossing for semi-autonomous vehicles with communication capacities. We design an intersection supervisor based on a mixed-integer quadratic programming approach which monitors the control inputs of each vehicle, and overrides those controls when necessary to ensure that all vehicles can navigate safely. Moreover, the solution control deviates minimally from the vehicles target inputs: overriding only occurs when it is strictly necessary, in which case the control is chosen as close as possible to the driver's intent. We theoretically prove that the supervisor needs only consider a finite future time horizon to ensure safety and deadlock avoidance over an infinite time horizon, and we demonstrate through simulation that this algorithm can work in real time. Additionally, unlike previous work, our formulation is suitable for complex intersection geometries with a high number of vehicles.
\end{abstract}

\section{INTRODUCTION}

While self-driving vehicles are not likely to reach consumer market before a few more years, most of the technology required for semi-autonomy already exists. For instance, adaptive cruise control [1] or advanced emergency braking [2] are becoming standard equipment in new cars to assist, and sometimes supplant, human drivers in order to improve safety and driving comfort. Such systems are efficient to prevent rear-end collisions, but they are not suited to more complex situations such as intersections, which represented more than $20 \%$ of road fatalities in the United States in 2014.

Until recently, most studies considered fully autonomous driving at intersections, assuming that vehicles were perfectly controlled [3], [4], and able to exchange their future intentions and to quickly and cooperatively react to any unexpected event. Some methods do exist to allow autonomous cars to handle unexpected maneuvers from human-driven vehicles [5], but the safety of the human-driven vehicle itself is rarely considered.

In this article, we consider a road intersection with semiautonomous vehicles equipped with communication capability, driving along fixed paths through the intersection (see,

1 MINES ParisTech, PSL Research University, Centre for robotics, 60 Bd St Michel 75006 Paris, France [florent.altche, xiangjun.qian, arnaud.de_la_fortelle]

emines-paristech.fr

2 École des Ponts ParisTech, Cité Descartes, 6-8 Av Blaise Pascal, 77455 Champs-sur-Marne, France

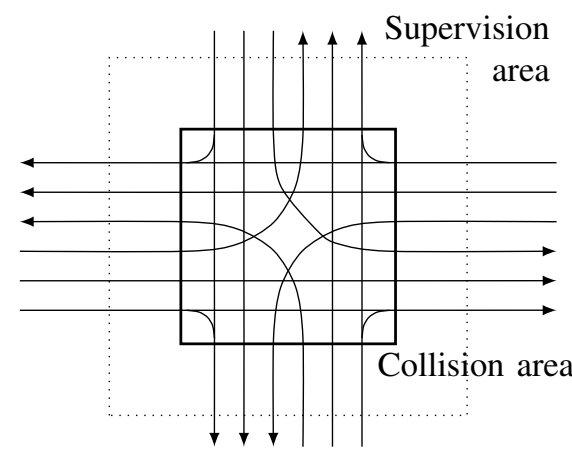

Fig. 1. Example of paths in a general intersection with three-lane roads. The outer (dotted) square is the boundary of the supervision area, and the inner square defines the collision area.

e.g., Fig. 1). At a given time, each vehicle is assumed to have a preferred desired control input for the next few fractions of a second: it can simply consist of tracking a desired speed or a more complicated human input. As vehicles are not fully autonomous, this target control may, or may not lead the system of vehicles into a so-called "inevitable collision state" [6], or in a deadlock configuration where no participant is able to cross the intersection. Therefore, an outside input is needed to ensure safe and non-blocking coordination of the vehicles across the intersection. Note that the fixed-paths assumption is classic in intersection problems, as vehicles generally drive inside a preassigned lane.

Colombo et al. [7] introduced the idea of a supervisory instance (called supervisor) tasked with preventing the system of vehicles from entering undesirable states by overriding the controls of one or several vehicles only when necessary. The question of determining whether overriding is needed or not, called "verification problem", is NP-hard [8]. Under several simplifying assumptions, this verification problem is shown in [7] to be equivalent to solving a scheduling problem, by assigning time slots during which only one vehicle is allowed inside the intersection. This allows to design a socalled least restrictive supervisor, which verifies the safety of the desired inputs and overrides them if necessary. However, the proposed supervisor only works with simple intersection geometries with one conflict point, i.e. only one vehicle can be inside the intersection at any given time. Moreover, no bound is given on the controls used for overriding, which can widely deviate from the desired ones. We call a supervisor minimally deviating if it minimizes the difference between the desired and overridden controls.

Several variations have been proposed based on the equiv- 
alence demonstrated in [7]. Reference [9] designs a supervisor which is robust to bounded uncertainties by adding safety margins. Reference [10] leverages job-shop scheduling to develop a supervisor that considers several possible conflict points inside the intersection; however, vehicles are modeled as single integrators which is not realistic in a realworld setting. Campos et al. [11] proposed a Pareto-optimal supervisor leading to a minimally deviating formulation by recursively finding the most constrained vehicle, reserving its optimal crossing time, and scheduling the crossing of the other vehicles using the previously scheduled ones as constraints. This method allows to minimize the deviation between the overridden and desired controls, but may be computationally intensive.

Inspired by previous work on using operations research for autonomous intersection management [12] and studies demonstrating the interest of using pairwise priorities between vehicles [13], we propose a novel design of a least restrictive and minimally deviating supervisor. Our main contribution is a new formulation of the supervisory problem as a finite horizon Model Predictive Control (MPC) problem, in which the safety constraints are modeled as mixedinteger linear inequalities on vehicles control and a quadratic objective function is used to penalize the deviation from the vehicles target control inputs. This formulation, extending our previous work on time-optimal coordination of fully autonomous vehicles [14], results in a mixed-integer quadratic program (MIQP) that can be solved using widely available software. Compared to previous approaches [7], [9], [10], the advantage of our formulation is twofold: 1) intersections with complex geometries (crossing, merging and diverging paths) can be handled intuitively, 2) the computational burden can be significantly reduced as we only consider a finite time horizon, and use highly efficient MIQP solvers.

The rest of this paper is articulated as follows. In Section II, we describe the modeling of the system of vehicles and, inspired by [7], [11], formally define a "Supervision problem" and its optimal, minimally deviating version. In Section III, we present an infinite horizon MPC formulation which solves the discretized optimal supervision problem. In Section IV, we deduce an equivalent, finite time horizon MPC problem which guarantees infinite horizon safety and deadlock avoidance. These results are demonstrated in Section V using computer simulation; finally, Section VI concludes the study.

\section{Problem Statement}

\section{A. System modeling}

We consider the problem of safely coordinating a set $\mathcal{N}$ of $N$ semi-autonomous vehicles at a generic road intersection, which could be comprised of multiple entry and exit lanes. Contrary to full autonomy, we assume that semi-autonomous vehicles are able to safely navigate outside intersections, for instance with adaptive cruise control techniques, but need a form of coordination to safely cross intersections. Therefore, we consider a finite supervision area comprising the intersection and a portion of the roads leading to, and exiting from the intersection, and only consider possible collisions inside this area. In the supervision area, vehicles are assumed to follow predetermined paths with good lateral accuracy. Fig. 1 shows an example intersection between two roads, each one comprising three entry and exit lanes, and the corresponding possible paths inside the intersection.

We model the longitudinal dynamics of each vehicle as a double integrator: for a vehicle $i \in \mathcal{N}$ following a path $\gamma_{i}$, the curvilinear position $s_{i}$, longitudinal speed $v_{i}$ and control input (or acceleration) $u_{i}$ are linked as

$$
\left(\begin{array}{c}
\dot{s}_{i} \\
\dot{v}_{i}
\end{array}\right)=\left(\begin{array}{c}
v_{i} \\
u_{i}
\end{array}\right) .
$$

We choose the origin of $s_{i}$ so that $s_{i}=0$ when the front bumper of vehicle $i$ enters the supervision area, and $s_{i}=$ $s_{i}^{\text {out }}>0$ when its rear bumper exits the supervision area. Vehicles are assumed to only move forward along their paths, so that for all $i \in \mathcal{N}, v_{i} \geq 0$. Furthermore, we consider bounds on the speed and acceleration of each vehicle, noted $v_{i} \in V_{i}=\left[0, \bar{v}_{i}\right]$ and $u_{i} \in U_{i}=\left[\underline{u}_{i}, \bar{u}_{i}\right]$ where $\underline{u}_{i}<0<$ $\bar{u}_{i}$. We let $x_{i}=\left(s_{i}, v_{i}\right)$ be the state of vehicle $i$, and we use the bold characters $\mathbf{x}=\left(x_{i}\right)_{i \in \mathcal{N}}$ and $\mathbf{u}=\left(u_{i}\right)_{i \in \mathcal{N}}$ to respectively denote the vectors of states and control inputs for the system of all vehicles.

We consider complex intersection geometries with merging, diverging, crossing and non-conflicting paths, in which both rear-end and side collisions may occur. For two vehicles $i, j$ with a risk of rear-end collision, we note $S_{i j}^{\|}=\left[\underline{s}_{i j}^{\|}, \bar{s}_{i j}^{\|}\right]$ the interval of positions $s_{i}$ where $i$ can have rear-end collisions with $j$. For vehicles on the same path, $S_{i j}^{\|}=\left[0, s_{i}^{\text {out }}\right]$; for vehicles on paths diverging at the curvilinear position $s_{i j}^{\text {diverge }}, S_{i j}^{\|}=\left[0, s_{i j}^{\text {diverge }}\right]$; for vehicles on merging paths, $S_{i j}^{\|}$is in the form $\left[s_{i j}^{\text {merge }}, s_{i j}^{\text {out }}\right]$. If there is no risk of rear-end collision for $i$ and $j$, we let $S_{i j}^{\|}=\emptyset$.

We call collision area the center of the intersection where side collisions can occur, as presented in Fig. 1. Note that side collisions cannot occur for vehicles on diverging paths, but can occur for merging paths. The portion of the path of a vehicle $i$ comprised in the collision area is called the collision segment for vehicle $i$, and we note $S_{i}^{\perp}=\left[\underline{s}_{i}^{\perp}, \bar{s}_{i}^{\perp}\right]$ the set of curvilinear coordinates corresponding to this segment. For two vehicles $i$ and $j$, we let $\mathcal{C}_{i j}$ be the set of positions $\left(s_{i}, s_{j}\right) \in S_{i}^{\perp} \times S_{j}^{\perp}$ where a side collision between $i$ and $j$ can happen, and we say that two vehicles $i$ and $j$ are conflicting if $\mathcal{C}_{i j} \neq \emptyset$. To ensure a safe crossing of the intersection, we require that two conflicting vehicles cannot be inside their collision segments simultaneously. However, to reduce unnecessary delays and increase traffic efficiency, non-conflicting vehicles are allowed to be inside their collision segments at the same time.

At a given time $\tau$, we say that the corresponding system state $\mathbf{x}(\tau)$ is safe if there exists a control $t \mapsto \mathbf{u}(t) \in$ $\prod_{i \in \mathcal{N}} U_{i}$ defined over $[\tau,+\infty$ [ corresponding to a safe infinite-horizon system trajectory $t \mapsto \mathbf{x}(t)$ over $[\tau,+\infty[$, i.e. for which no rear-end or side collision happens at any future time. On the contrary, if no such control exists, the 
state $\mathbf{x}(\tau)$ is called an inevitable collision state [6]. Formally, an infinite horizon trajectory is safe if, $\forall t \geq \tau, \forall i, j \in \mathcal{N}$ :

$$
\begin{aligned}
\mathcal{C}_{i j} \neq \emptyset \Rightarrow\left(s_{i}(t), s_{j}(t)\right) \notin S_{i}^{\perp} \times S_{j}^{\perp} \\
\left(s_{i}(t), s_{j}(t)\right) \in S_{i j}^{\|} \times S_{j i}^{\|} \Rightarrow \\
\quad\left(s_{i}(t) \geq s_{j}(t)+d_{i j} \vee s_{j}(t) \geq s_{i}(t)+d_{j i}\right)
\end{aligned}
$$

where $d_{i j}$ and $d_{j i}$ account for a safe following distance and a potential offset of longitudinal positions between vehicles. Note that condition (3) corresponds to either $i$ following $j$ or $j$ following $i$, accounting for the case of vehicles on merging paths, for which a relative order is not set in advance.

\section{B. Discretized dynamics}

At a given time, each vehicle has a certain desired control $u_{i, d e s}$, for instance taking into account passengers comfort, energy consumption or time constraints. The vector of these desired controls defines a desired system control $\mathbf{u}_{\text {des }}$. Depending on the dynamic constraints and the current system state, this desired control may lead the system into an unsafe situation, i.e. a state where a collision is inevitable.

Following the ideas introduced in [7], we define a Supervisor as an instance working in discrete time, which assigns a control to each vehicle to ensure that the system remains in a safe state for all future times. We let $\Delta t>0$ be the duration of a time step for the supervisor, and we assume that individual vehicles update their control in discrete time with the same time step duration, so that the dynamic equations for a vehicle $i$ can be rewritten as:

$$
\begin{aligned}
& s_{i}^{k+1}=v_{i}^{k} \Delta t+\frac{1}{2} u_{i}^{k} \Delta t^{2} \\
& v_{i}^{k+1}=v_{i}^{k}+u_{i}^{k} \Delta t
\end{aligned}
$$

where $s_{i}^{k}, v_{i}^{k}$ and $u_{i}^{k}$ are respectively the position, speed and control of the vehicle at the beginning of the time interval $\left[k \Delta t,(k+1) \Delta t\left[\right.\right.$. We note $x_{i}^{k}=\left(s_{i}^{k}, v_{i}^{k}\right)$ the state of a single vehicle, and we let $\mathbf{x}^{k}=\left(x_{i}^{k}\right)_{i \in \mathcal{N}}$ and $\mathbf{u}^{k}=\left(u_{i}^{k}\right)_{i \in \mathcal{N}}$.

As in the continuous case, we formally introduce the safety of the discretized system: at a time instant $\kappa$, we say that the current system state $\mathbf{x}^{\kappa}$ is safe if there exists a piecewiseconstant control $\left(\mathbf{u}^{k}\right)_{k \geq \kappa}$ that ensures the existence of a safe infinite-horizon system trajectory $\left(\mathbf{x}^{k}\right)_{k \geq \kappa}$. Note that directly discretizing conditions (2) and (3) is not sufficient to ensure the infinite horizon safety of the discretized system. Therefore, we adopt the following sufficient conditions for system safety, adapted from (2) and (3), and we require that for all $k \geq \kappa$ and all $i, j \in \mathcal{N}$,

$$
\begin{gathered}
\left(\mathcal{C}_{i j} \neq \emptyset \wedge s_{i}^{k} \in S_{i}^{\perp}\right) \Rightarrow s_{j}^{k+1} \notin S_{j}^{\perp} \\
\left(s_{i}^{k}, s_{j}^{k}\right) \in S_{i j}^{\|} \times S_{j i}^{\|} \Rightarrow \\
\left(s_{i}^{k+1} \geq s_{j}^{k+1}+d_{i j} \vee s_{j}^{k+1} \geq s_{i}^{k+1}+d_{j i}\right) \\
\left(\left(s_{i}^{k}, s_{j}^{k}\right) \in S_{i j}^{\|} \times S_{j i}^{\|} \wedge s_{i}^{k+1} \geq s_{j}^{k+1}+d_{i j}\right) \Rightarrow \\
s_{i}^{k+1}-s_{j}^{k+1}+\frac{\Delta t}{2}\left(v_{i}^{k+1}-v_{j}^{k+1}\right) \geq d_{i j}
\end{gathered}
$$

The additional condition (8) ensures the absence of longitudinal collisions between two time steps.

\section{Supervision problem}

At the beginning of a time step $\kappa$ and for an initially safe system state $\mathbf{x}^{\kappa}$, we note $\mathbf{u}_{\text {des }}^{\kappa}$ the desired (constant) system control over $[\kappa \Delta t,(\kappa+1) \Delta t[$. The Supervisor is tasked with returning a safe (constant) control $\mathbf{u}_{\text {safe }}^{\kappa}$ for the set of vehicles so that the next system state $\mathbf{x}^{\kappa+1}$ is safe. In what follows, we note $\mathcal{U}_{\text {safe }}^{\kappa} \subset \prod_{i} U_{i}$ the set of safe constant system controls over $[\kappa \Delta t,(\kappa+1) \Delta t[$.

As introduced in [7] we define the (least restrictive) Supervision Problem $(S P)$ as follows:

Problem 1: $S P$. For a safe system state $\mathbf{x}^{\kappa}$ and a desired system control $\mathbf{u}_{d e s}^{\kappa}$, return $\mathbf{u}_{\text {safe }}^{\kappa}=S P\left(\mathbf{x}^{\kappa}, \mathbf{u}_{\text {des }}^{\kappa}\right)$ such that $\mathbf{u}_{\text {safe }}^{\kappa} \in \mathcal{U}_{\text {safe }}^{\kappa}$ and so that $\mathbf{u}_{\text {safe }}^{\kappa}=\mathbf{u}_{\text {des }}^{\kappa}$ if $\mathbf{u}_{\text {des }}^{\kappa} \in \mathcal{U}_{\text {safe }}^{\kappa}$.

In the supervised driving paradigm, the control actually implemented by the vehicles over $\left[\kappa \Delta t,(\kappa+1) \Delta t\left[\right.\right.$ is $\mathbf{u}_{\text {safe }}^{\kappa}$. The supervision problem $S P$ is least restrictive in the sense that an "overriding" only occurs when the desired system input would steer the system into an inevitable collision state. The fact that the state reached using $\mathbf{u}_{s a f e}^{\kappa}$ is safe ensures the recursive feasibility of $S P$.

We now define the optimal Supervision Problem $\left(S P^{*}\right)$, which is a generalization of [11]. We say that a supervisor solving $S P^{*}$ is a minimally deviating supervisor for a given norm $\|\cdot\|$ over the set of bounded system controls

Problem 2: $S P^{*}$. For a system state $\mathbf{x}^{\kappa}$ and a desired system control $\mathbf{u}_{d e s}^{\kappa}$, return $\mathbf{u}_{\text {safe }}^{* \kappa}=S P^{*}\left(\mathbf{x}^{\kappa}, \mathbf{u}_{d e s}^{\kappa}\right)$ such that $\mathbf{u}^{* \kappa}$ safe $=\arg \min _{\mathbf{u} \in \mathcal{U}_{\text {safe }}^{\kappa}}\left\|\mathbf{u}^{\kappa}-\mathbf{u}_{\text {des }}^{\kappa}\right\|$.

Note that the optimal supervision problem is an instance of the least-restrictive supervision problem, in the sense that any solution to $S P^{*}$ is a solution to $S P$.However, not all solutions of $S P$ are solutions of $S P^{*}$. Previous literature [7], [9], [10] mainly considers $S P$, by converting it to an equivalent scheduling problem. Campos et al. [11] proposed to exhaustively enumerate all solutions of $S P$ to find the solution to $S P^{*}$, which may be computationally costly. In this paper, we propose an MPC-based approach that can solve $S P^{*}$ efficiently, even with a large number of vehicles and complex intersection geometries.

\section{INFINITE HORIZON MPC FORMULATION FOR $S P^{*}$}

In this section, we present an infinite horizon MPC formulation to exactly solve the optimal supervision problem $S P^{*}$.

For two conflicting vehicles $i$ and $j$, we introduce the binary decision variable $\pi_{i j}$ which is equal to 1 if $i$ goes before $j$, and 0 otherwise; we require that $\pi_{i j}+\pi_{j i}=1$. Moreover, we define a set of indicator variables $\varepsilon$ such that $\varepsilon_{i}^{\perp, i n}(k)=1$ if $s_{i}^{k} \geq \underline{s}_{i}^{\perp}$ and $\varepsilon_{i}^{\perp, \text { out }}(k)=1$ if $s_{i}^{k} \geq \bar{s}_{i}^{\perp}$. Similarly, we let $\varepsilon_{i j}^{\|, i n}(\bullet, k)=1$ if $s_{\bullet}^{k} \geq \underline{s}_{i j}^{\|}(\bullet)$ and $\varepsilon_{i j}^{\|, \text {out }}(\bullet, k)=1$ if $s_{\bullet}^{k} \geq \bar{s}_{i j}^{\|}(\bullet)$ for $\bullet=i$ or $j$. Reference [14] shows how constraints (2) and (3), as well as the constraints on the indicator variables $\varepsilon$ can be enforced using linear inequalities involving continuous and integer variables using a "big-M" formulation. To ensure safety and deadlock avoidance over an infinite time horizon we require, for every pair of conflicting vehicles $i$ and $j$ and for all time 
steps $k$ :

$$
\begin{gathered}
\pi_{i j}=1 \wedge \varepsilon_{i}^{\perp, \text { out }}(k)=0 \Rightarrow \varepsilon_{j}^{\perp, \text { in }}(k+1)=0 \\
\pi_{i j}=1 \wedge \varepsilon_{j i}^{\|, \text {in }}(k)=1 \wedge \varepsilon_{i j}^{\|, \text {out }}(k)=0 \Rightarrow \\
s_{i}^{k+1}-s_{j}^{k+1} \geq d_{i j} \\
\pi_{i j}=1 \wedge \varepsilon_{j i}^{\|, \text {in }}(k)=1 \wedge \varepsilon_{i j}^{\|, \text {out }}(k)=0 \Rightarrow \\
s_{i}^{k+1}-s_{j}^{k+1}+\frac{\Delta t}{2}\left(v_{i}^{k+1}-v_{j}^{k+1}\right) \geq d_{i j}
\end{gathered}
$$

Note that (s1), (s2) and (s3) respectively correspond to (6), (7) and (8).

Conditions (s2) and (s3) are used for vehicles on merging paths. For non-conflicting vehicles having following constraints (i.e. on parallel or diverging paths), similar conditions are required but the value of $\pi_{i j}$ is implicitly chosen based on the initial positions of the vehicles.

Any trajectory verifying the above conditions (for all vehicles for which they are applicable) at all time steps is safe over an infinite time horizon. To ensure trajectories are dynamically feasible for the vehicles, we also enforce constraints (4) and (5), as well as bound constraints $v_{i}^{k} \in$ $\left[0, \bar{v}_{i}\right]$ and $u_{i}^{k} \in\left[\underline{u}_{i}, \bar{u}_{i}\right]$ for all vehicles and at all time steps. As a result, any control verifying all the above conditions is a member of $\mathcal{U}_{\text {safe }}^{\kappa}$.

To choose the optimal control (in the sense of $S P^{*}$ ) in $\mathcal{U}_{\text {safe }}^{\kappa}$, we introduce the following objective function:

$$
\mathcal{O}_{\kappa}=\sum_{i \in \mathcal{N}} w_{i}^{\kappa}\left(u_{i, d e s}^{\kappa}-u_{i}^{\kappa}\right)^{2}
$$

where the constant $w_{i}^{\kappa} \geq 0$ is a weighting coefficient for vehicle $i$ at step $\kappa$ which can be used, for instance, to distinguish high priority vehicles. From the previous considerations and noting $\mathbf{X}=\left(X^{k}\right)_{k \geq \kappa}$ where $X^{k}$ is the tuple of the variables defined above at step $k$, we deduce the following theorem about our Infinite Horizon Model Predictive Control (IH-MPC) formulation:

Theorem 1: Let $\mathbf{u}^{\kappa}$ be a solution of the optimization problem:

$$
\begin{aligned}
& \mathbf{u}^{\kappa}=\Pi_{\mathbf{u}} \underset{\mathbf{X}}{\arg \min } \quad \mathcal{O}_{\kappa}(\mathbf{X}) \\
& \text { subj. to } \forall k \geq \kappa,(4),(5),(\mathrm{s} 1)-(\mathrm{s} 3)
\end{aligned}
$$

(IH-MPC)

where $\Pi_{\mathbf{u}}$ is a projection operator consisting of only keeping the components of the solution corresponding to $\mathbf{u}^{\kappa}$. Then $\mathbf{u}^{\kappa}$ is a solution to the optimal supervision problem $S P^{*}$ at step $\kappa$ for a (semi-)norm of $\mathbb{R}^{N}$.

Proof: We note that any solution to the above problem ensures safety for all vehicles at all future times; therefore, the corresponding control $\mathbf{u}^{\kappa}$ is in $\mathcal{U}_{\text {safe }}^{\kappa}$ and is thus a solution to $S P$. Moreover, by construction the solution minimizes $\mathcal{O}_{\kappa}$, thus it is a solution to $S P^{*}$ for the seminorm associated with the bilinear form $\Pi_{\mathbf{u}} \mathcal{O}_{\kappa}$. Note that, if all $w_{i}^{\kappa}=1$, this corresponds to the euclidean norm of $\mathbb{R}^{N}$.

One property of this receding horizon formulation is the guarantee that all vehicles can eventually exit the supervision area, and that no deadlock situation can happen, as stated in the following theorem:

Theorem 2: For all $\kappa$, there exists a feasible solution to IH-MPC in which all vehicles in $\mathcal{N}$ exit the supervision area in finite time.

Proof: The proof builds on previous results from [13], defining the notion of "priority graph" for a set of vehicles. For a valuation of the priority variables $\pi_{i j}$, the corresponding priority graph is built by choosing each vehicle as a vertex, and adding the (directed) edge $i \rightarrow j$ if and only if $\pi_{i j}=1$. It is shown in [13] that no deadlock can arise from an acyclic priority graph; this corresponds to the intuitive notion that deadlocks only occur in the presence of circular wait lists.

Assume that some vehicles are in a deadlock situation: by definition, there exists a time step $k \geq \kappa$ when all deadlocked vehicles are stopped; moreover, the above results imply that the vehicles have been assigned cyclic priorities. The problem constraints ensure that, for any pair of vehicles $(i, j)$ involved in the circular wait list with assigned priority $\pi_{i j}=1$, we necessarily have $s_{i}^{k} \leq \underline{s}_{i}^{\perp}$ and $s_{j}^{k} \leq \underline{s}_{j}^{\perp}$. Therefore, assigning priority $\pi_{j i}=1$ is also feasible, and thus it is possible to reverse the edge $i \rightarrow j$ in the priority graph without violating any constraint.

Results on minimum feedback arc sets [15] show that it is possible to remove cycles in a directed graph by reversing a set of its edges, all of these edges participating in at least one cycle. This property ensures that, if all vehicles involved in circular wait lists are stopped at step $k$, there exists a feasible solution with acyclic priorities which proves the theorem.

\section{An equivalent Finite Horizon formulation}

In the previous section, we introduced an optimal and least restrictive supervisor based on an infinite horizon MPC scheme. However, this formulation is not suitable for practical implementations because of the infinite number of variables. We now present a finite horizon formulation which can be implemented in widely available solvers and, given the proper choice of the time horizon, ensures the safety of the system over an infinite time horizon.

\section{A. Finite Horizon formulation}

We propose an approach based on a restriction of the infinite horizon formulation introduced in the previous section. At a time step $\kappa$, we consider the restriction of IH-MPC to the first following $K$ steps (corresponding to a horizon $T=K \Delta t$ ), i.e. $\mathcal{O}_{\kappa}$ is minimized under constraints (4), (5) and (s1) to (s3) for all steps $k$ such that $\kappa \leq k \leq \kappa+K$, and the last considered variables are those at step $k+K$. We note FH-MPC this finite horizon Model Predictive Control problem.

\section{B. Equivalence of FH-MPC and IH-MPC}

By construction, a solution to FH-MPC is guaranteed to avoid collisions up to time $(\kappa+K) \Delta t$. However, due to the dynamic constraints on the vehicles, there is no a priori guarantee that the resulting trajectory can be extended to a 
safe infinite horizon trajectory, i.e. $\mathbf{x}^{\kappa+1}$ might not be safe. In what follows, we prove that provided the horizon $T=K \Delta t$ is chosen long enough, the solution $\mathbf{u}^{\kappa}$ of FH-MPC ensures that $\mathbf{x}^{\kappa+1}$ is safe.

First, note that the constraints in FH-MPC are a restriction of those of IH-MPC. Therefore, the restriction of any solution to IH-MPC to the interval $[\kappa \Delta t,(\kappa+K) \Delta t]$ is a feasible (but potentially not optimal) solution of FH-MPC, and so:

Proposition 1: If there exists a solution to IH-MPC, then the restriction of the solution to the first $K$ steps is a feasible solution to FH-MPC.

We will now prove a reciprocal implication to Proposition 1, which is stated in Theorem 3. In what follows, we let $v_{\max }>0, u_{a}>0$ and $u_{b}<0$ be respectively a global upper bound for $\bar{v}_{i}$, lower bound for $\bar{u}_{i}$ and upper bound for $\underline{u}_{i}$ such that for all $i, \bar{v}_{i} \leq v_{\max }, \bar{u}_{i} \geq u_{a}$ and $\underline{u}_{i} \leq u_{b}$.

The key idea of the proof lies in the choice of a planning horizon long enough to allow any vehicle to fully stop. The structure of the proof is as follows: Lemma 1 gives a lower bound on the time horizon to allow a single vehicle to stop using discrete dynamics, although with a potential risk of rear-end collisions from following vehicles. In Proposition 2, we show that this time horizon is sufficient to ensure safety from lateral (but not necessarily from rear-end) collisions. Finally, in Proposition 3, we give a slightly higher bound on the time horizon ensuring that all vehicles in a line can all safely stop without rear-end collisions, and therefore that this horizon also ensures absence of rear-end collisions.

Lemma 1: Let $\Delta t>0$ be a time step duration and consider a horizon $T=K \Delta t \geq \frac{v_{\max }}{\left|u_{b}\right|}+\Delta t$ at time $t_{1}=\kappa \Delta t$. Consider a vehicle $i$ for which there exists a discrete control $\left(u_{i}^{k}\right)_{\kappa \leq k<\kappa+K}$ such that, for all $\kappa \leq k<\kappa+K, u_{i}^{k} \in\left[\underline{u}_{i}, \bar{u}_{i}\right]$ corresponding to a dynamically feasible trajectory $s_{i}(t)$ over $[\kappa \Delta t,(\kappa+K) \Delta t]$.

There exists a discrete control $\left(\tilde{u}_{i}^{k}\right)_{\kappa \leq k \leq \kappa+K}$ such that for all $\kappa \leq k \leq \kappa+K, u_{i}^{k} \in\left[\underline{u}_{i}, \bar{u}_{i}\right]$ and $\tilde{u}_{i}^{\kappa}=u_{i}^{\kappa}$, and for which the corresponding dynamically feasible trajectory $t \mapsto$ $\tilde{x}_{i}(t)=\left(\tilde{s}_{i}(t), \tilde{v}_{i}(t)\right)$ verifies $\tilde{s}_{i}\left(t_{1}+T+\Delta t\right) \leq s_{i}\left(t_{1}+T\right)$ and $\tilde{v}_{i}=0$ over $\left[t_{1}+T, t_{1}+T+\Delta t\right]$.

Proof: Let $\left(u_{i}^{k}\right)$ be the control corresponding to trajectory $s_{i}$, and let us define a control $\left(w_{i}^{k}\right)$ as: $w_{i}^{\kappa}=u_{i}^{\kappa}$, $w_{i}^{k}=\min \left(u_{b}, u_{i}^{k}\right)$ for $\kappa<k<\kappa+K$, and $w_{i}^{\kappa+K}=u_{b}$. We construct $\left(\tilde{u}_{i}^{k}\right)$ iteratively as $\tilde{u}_{i}^{\kappa}=u_{i}^{k}$ and, for $k \geq \kappa+1$, $\tilde{u}_{i}^{k}=\left\{\begin{array}{ll}w_{i}^{k} & \text { if } \tilde{v}_{i}^{k}+w_{i}^{k} \Delta t \geq 0 \\ -\frac{v_{i}^{k}}{\Delta t} & \text { otherwise }\end{array}\right.$, where $\tilde{v}_{i}^{k}$ is the speed of vehicle $i$ at time $k \Delta t$ under control $\left(\tilde{u}_{i}^{k}\right)$.

As $\tilde{v}_{i}^{\kappa+1}=v_{i}^{\kappa+1} \leq v_{\max } \leq(K-1) \Delta t\left|u_{b}\right|$ from the hypothesis, there exists a minimal value of $k_{0} \geq \kappa$ such that $\tilde{v}_{i}^{k_{0}} \leq\left|u_{b}\right| \Delta t$; moreover, the condition on $K$ ensures that $\tilde{v}_{i}^{\kappa+1}-(K-2)\left|u_{b}\right| \Delta_{t} \leq\left|u_{b}\right| \Delta_{t}$, and so $k_{0} \leq(\kappa+1)+$ $(K-2)=\kappa+K-1$.

From the definition of $\left(\tilde{u}_{i}^{k}\right)$, we know that for all $k_{0}+1 \leq$ $k \leq \kappa+K, \tilde{v}_{i}^{k}=0$. Since $\tilde{u}_{i}^{k} \leq u_{i}^{k}$ for $\kappa \leq k \leq k_{0}-1$, we also know that $\tilde{s}_{i}^{k_{0}} \leq s_{i}^{k_{0}}$ and $\tilde{v}_{i}^{k_{0}} \leq v_{i}^{k_{0}}$. Finally, $\tilde{u}_{i}^{k_{0}}$ is the minimal admissible control starting from $\tilde{x}_{i}^{k_{0}}$; therefore, $\tilde{s}_{i}^{k_{0}+1} \leq s_{i}^{k_{0}+1}=s_{i}^{\kappa+K}$ which proves the above lemma.
We can now formulate the following proposition, in the particular case where no pair of vehicles has following constraints:

Proposition 2: Let $\Delta t>0$ and $T=K \Delta t \geq \frac{v_{\max }}{\left|u_{b}\right|}+\Delta t$. Suppose that there exists a solution of FH-MPC at step $\kappa$ leading the system of vehicles in $\mathcal{N}$ in a state $\mathbf{x}^{\kappa+1}$ at step $\kappa+1$, and that vehicles have no following constraints.

There exists a solution of FH-MPC at step $\kappa+1$ starting from state $\mathbf{x}^{\kappa+1}$ with horizon $T$.

Proof: We will show that, using control $\mathbf{u}^{\kappa}$ over $[\kappa \Delta t,(\kappa+1) \Delta t[$, the safe crossing constraints (s1) of FHMPC remain feasible at step $\kappa+K+1$ without violating the kinodynamic constraints (4) and (5).

First, if $s_{i}^{\kappa+1} \geq \bar{s}_{i}^{\perp}$ for all $i \in \mathcal{N}$, the crossing constraints are trivially feasible for $\kappa \leq k \leq \kappa+K+1$. Otherwise, let $i$ be a vehicle such that $s_{i}^{\kappa+1} \leq \bar{s}_{i}^{\perp}$. If $s_{i}^{\kappa+K} \leq \underline{s}_{i}^{\perp}$, proposition 3 shows that $i$ can fully stop before reaching $\underline{s}_{i}^{\perp}$ and so the crossing constraints involving $i$ remain feasible. If $\underline{s}_{i}^{\perp} \leq s_{i}^{\kappa+K} \geq \bar{s}_{i}^{\perp}$, condition (s1) ensures that each vehicle $j$ conflicting with $i$ either verifies $s_{j}^{\kappa+K} \leq \underline{s}_{j}^{\perp}$ (in which case $j$ can fully stop), or $s_{j}^{\kappa+K} \geq \bar{s}_{j}^{\perp}$ (in which case constraint (s1) is always satisfied). Finally, if $s_{i}^{\kappa+K} \geq$ $\bar{s}_{i}^{\perp}$, crossing constraints involving $i$ are inactive and thus satisfied. In all cases, the safe crossing constraints remain feasible.

When following constraints are involved, the condition on the time horizon becomes more restrictive. In the following proposition and noting $\lceil\cdot\rceil$ the ceiling function, we prove a bound ensuring that a line of vehicles can safely stop before the leader reaches its final computed position at the end of the time horizon, without risk of rear-end collisions:

Proposition 3: Let $\Delta t>0$ and suppose that $p$ vehicles (noted $1, \ldots, p$ from rear to front) are following one another at step $\kappa$. We consider a horizon $T=K \Delta t \geq \frac{v_{\max }}{\left|u_{b}\right|}+$ $(p-1)\left(1+\left\lceil\frac{u_{\max }}{\left|u_{b}\right|}\right\rceil\right) \Delta t+\Delta t$ at time $t_{1}=\kappa \Delta t$, and we assume that every vehicle $i \in\{1, \ldots, p\}$ has a safe discrete control $\left(u_{i}^{k}\right)_{\kappa \leq k<\kappa+K}$ such that, for all $\kappa \leq k<\kappa+K$, $u_{i}^{k} \in\left[\underline{u}_{i}, \bar{u}_{i}\right]$. We let $t \mapsto x_{i}(t)$ be the safe trajectory over $\left[t_{1}, t_{1}+T\right]$ for vehicle $i$ under control $\left(u_{i}^{k}\right)$.

For all $i \in\{1, \ldots, p\}$, there exists a safe discrete control $\left(\hat{u}_{i}^{k}\right)_{\kappa \leq k \leq \kappa+K}$ such that for all $\kappa \leq k \leq \kappa+K, u_{i}^{k} \in\left[\underline{u}_{i}, \bar{u}_{i}\right]$, $\hat{u}_{i}^{\kappa}=u_{i}^{\kappa}$ and for which the corresponding dynamically feasible and safe trajectory $t \mapsto \hat{x}_{i}(t)=\left(\hat{s}_{i}(t), \hat{v}_{i}(t)\right)$ verifies $\hat{s}_{i}\left(t_{1}+T+\Delta t\right) \leq s_{i}\left(t_{1}+T\right)$ and $\hat{v}_{i}=0$ over $\left[t_{1}+T, t_{1}+T+\Delta t\right]$.

Proof: We will prove by induction that, for $i \in$ $\{1, \ldots, p\}$, there exists a dynamically feasible control $\left(\hat{u}_{i}^{k}\right)$ with $\hat{u}_{i}^{\kappa}=u_{i}^{\kappa}$ and $\hat{u}_{i}^{k} \leq u_{i}^{k}$ for $k \geq \kappa$ such that the corresponding vehicle speed $\left(\hat{v}_{i}^{k}\right)$ verifies $\hat{v}_{i}^{\kappa+K_{i}} \leq\left|u_{b}\right| \Delta t$ with $K_{i} \Delta t=\left\lceil\frac{v_{\max }}{\left|u_{b}\right|}\right\rceil+(i-1)\left(1+\left\lceil\frac{u_{\max }}{\left|u_{b}\right|}\right\rceil\right) \Delta t$. First, for the rearmost vehicle $i=1$, the proof of lemma 1 provides the result with $\hat{u}_{i}^{k}=\tilde{u}_{i}^{k}$.

We now let $i \geq 2$ and assume that every vehicle $j \in$ $\{1, \ldots, i-1\}$ follows its corresponding control $\left(\hat{u}_{j}^{k}\right)$. We note $\hat{s}_{j}^{k}$ and $\hat{v}_{j}^{k}$ the position and speed of vehicle $j$ at step $k$ under this control. Since $\hat{u}_{j}^{k} \leq u_{j}^{k}$ for these vehicles, we 
deduce from the monotony of the system that the original control solution for vehicle $i\left(u_{i}^{k}\right)_{\kappa \leq k<\kappa+K}$ prevents rearend collisions if vehicle $i-1$ applies $\left(u_{i-1}^{k}\right)$. Therefore, any dynamically feasible extension of $\left(u_{i}^{k}\right)$ is safe over $\left[\kappa \Delta t,(\kappa+K+1) \Delta t\left[\right.\right.$. As a result, the set $U_{i}^{\text {safe }}\left(u_{i}^{\kappa},[\kappa, \kappa+\right.$ $K]$ ) of all admissible controls $\left(\hat{u}_{i}^{k}\right)_{\kappa \leq k \leq \kappa+K}$ for vehicle $i$ such that $\hat{u}_{i}^{\kappa}=u_{i}^{\kappa}$ and $\underline{u}_{i}^{k} \leq \hat{u}_{i}^{k} \leq u_{i}^{k}$ for $\kappa \leq k<\kappa+K$ is not empty. We note $\left(\hat{u}_{i}^{k}\right)$ a minimum element of this set (and so $\hat{u}_{i}^{k} \leq u_{i}^{k}$ ); we will prove that $\hat{v}_{i}^{\kappa+K_{i}} \leq\left|u_{b}\right| \Delta t$.

If for all $k \geq \kappa, \hat{v}_{i}^{k} \leq \tilde{v}_{i-1}^{k}$, we conclude that vehicle $i$ stops before vehicle $i-1$ which proves the result from the induction hypothesis. Otherwise, we let $k_{0}^{i} \geq \kappa$ be the minimum $k$ such that $\hat{v}_{i}^{k} \geq \tilde{v}_{i-1}^{k}$, and we know that $\hat{v}_{i}^{k_{0}^{i}} \leq \hat{v}_{i-1}^{k_{0}^{i}-1}+u_{\max } \Delta t$. For all $k \geq k_{0}^{i}$, we know from the monotony of the system that the control $\min \left(\hat{u}_{i-1}^{k}, u_{i}^{k}, u_{b}\right)$ prevents rear-end collisions; we deduce that, for $k \geq k_{0}^{i}$, $\hat{v}_{i}^{k} \leq \hat{v}_{i-1}^{k-1}+u_{\max } \Delta t$. Therefore, $\hat{v}_{i}^{\kappa+K_{i-1}+1} \leq \hat{v}_{i-1}^{\overline{K_{i-1}}}+$ $u_{\max } \Delta t$ and we deduce from the induction hypothesis that $\hat{v}_{i}^{\kappa+K_{i-1}+1} \leq u_{\max } \Delta t+\left|u_{b}\right| \Delta t$. Therefore, we obtain the recursion relation $K_{i}=K_{i-1}+1+\left\lceil\frac{u_{\max }}{\left|u_{b}\right|}\right\rceil$ which yields the announced result.

Finally, we conclude that vehicle $i$ can fully stop (without rear-end collisions) at step $\kappa+K_{i}+1$; therefore the set of $p$ vehicles can safely stop before the beginning of step $\kappa+K$ if $K \geq K_{p}=\frac{v_{\max }}{\left|u_{b}\right| \Delta t}+(p-1)\left\lceil\frac{u_{\max }}{\left|u_{b}\right|}\right\rceil+1$. Since the recursion ensures that for all $i$ and $k, \hat{u}_{i}^{k} \leq u_{i}^{k}$, we deduce that $s_{i}^{\kappa+K} \leq \hat{s}_{i}^{\kappa+K}$ which proves the proposition.

Finally, we deduce the following theorem:

Theorem 3: Let $\Delta t>0$ and suppose that at most $p$ vehicles are following one another, and consider a horizon $T=K \Delta t \geq \frac{v_{\max }}{\left|u_{b}\right|}+(p-1)\left(1+\left\lceil\frac{u_{\max }}{\left|u_{b}\right|}\right\rceil\right) \Delta t+\Delta t$. At every time step $\kappa$, formulation IH-MPC is equivalent to FH-MPC with horizon $T$, i.e. the restriction of an optimal solution from one formulation can be extended to an optimal solution for the other in the sense of $\mathcal{O}_{\kappa}$.

Proof: Consider an optimal solution $\mathbf{u}_{\infty}$ to IH-MPC and an optimal solution $\mathbf{u}_{T}$ to FH-MPC. From proposition 1, we know that the restriction of $\mathbf{u}_{\infty}$ is a solution to FH-MPC, and therefore $\mathcal{O}_{\kappa}\left(\mathbf{u}_{\infty}\right) \geq \mathcal{O}_{\kappa}\left(\mathbf{u}_{T}\right)$ since $\mathbf{u}_{T}$ is optimal for FH-MPC. Moreover, from propositions 2 and 3, we know that $\mathbf{u}_{T}$ can be extended into an infinite horizon solution $\mathbf{u}_{T, \infty}$ by recursively solving the problem over successive time steps. Therefore, $\mathcal{O}_{\kappa}\left(\mathbf{u}_{T, \infty}\right) \geq \mathcal{O}_{\kappa}\left(\mathbf{u}_{\infty}\right)$; since $\mathcal{O}_{\kappa}$ only depends on the first time step of the solution, we deduce $\mathcal{O}_{\kappa}\left(\mathbf{u}_{T, \infty}\right)=\mathcal{O}_{\kappa}\left(\mathbf{u}_{T}\right)$ and so $\mathcal{O}_{\kappa}\left(\mathbf{u}_{\infty}\right)=\mathcal{O}_{\kappa}\left(\mathbf{u}_{T}\right)$.

Note that, from the previous equivalence, the liveness guarantee demonstrated in theorem 2 for the infinite horizon formulation also applies to the finite horizon one.

\section{Simulation Results}

The use of the FH-MPC formulation to design an optimal supervisor has been validated by computer simulation on the example of the intersection of Fig. 1. The simulation is based on the commercial MIQP solver Gurobi [16] and the free traffic modeling tool SUMO [17], and uses SUMO's path generation algorithm to compute collision segments

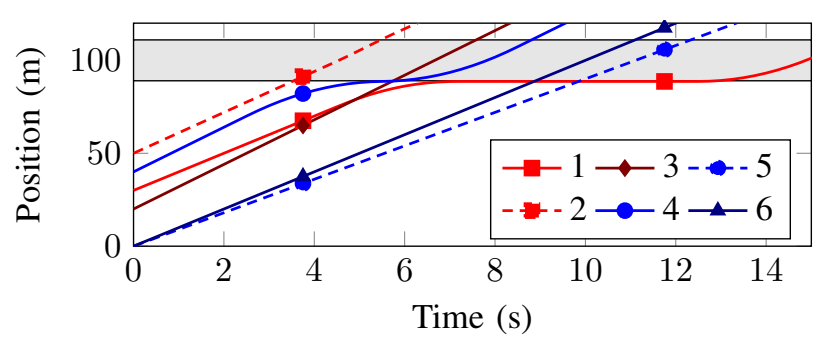

Fig. 2. Curvilinear positions of the supervised vehicles.

and conflicting vehicles. Simulations were performed on a personal computer running on a $3.60 \mathrm{GHz}$ Intel Core i7-4790 CPU with $16 \mathrm{~GB}$ of RAM.

In all simulations, vehicles are modeled as rectangles of $5 \mathrm{~m}$ length by $2 \mathrm{~m}$ width, with $\left[\underline{u_{i}}, \overline{u_{i}}\right]=[-4,+4] \mathrm{m} \mathrm{s}^{-2}$. The duration of a time step is chosen as $\Delta t=0.25 \mathrm{~s}$, and the horizon $T=4 \mathrm{~s}$, ensuring that the hypotheses of theorems 2 and 3 are verified. All weighting terms $w_{i}^{\kappa}$ are chosen as $w_{i}^{\kappa}=1$.

\section{A. Microscopic simulation}

To illustrate the safety and liveness of the proposed supervisor, we first provide a simple example of 6 vehicles in the intersection presented in Fig. 1. For clarity reasons, vehicles either come from the west or the south. Table I presents the parameters used for the first simulation (in meters and meters per second); note that vehicles 1 and 2 traveling from west to east and vehicles 4 and 5 traveling from south to north do so on the middle lane of the road, so that they do not conflict with vehicle 3 , which turns right. For $i \in\{1,2,4,5,6\}$, $\underline{s}_{i}^{\perp}=89 \mathrm{~m}$ and $\bar{s}_{i}^{\perp}=111 \mathrm{~m}$.

TABLE I

PARAMETERS FOR THE FIRST SIMULATION

\begin{tabular}{lllllll}
\hline$i$ & 1 & 2 & 3 & 4 & 5 & 6 \\
\hline Orig & West & West & West & South & South & South \\
Dest. & East & East & South & North & North & West \\
$s_{i}^{0}(\mathrm{~m})$ & 30 & 50 & 20 & 40 & 0 & 0 \\
$v_{i}^{0}\left(\mathrm{~m} \mathrm{~s}^{-1}\right)$ & 10 & 11 & 9 & 12 & 9 & 10 \\
\hline
\end{tabular}

Vehicles request a control corresponding to tracking their initial speed, with $u_{i, d e s}^{\kappa}=\frac{1}{\Delta t}\left(v_{i}^{0}-v_{i}^{\kappa}\right)$. Fig. 2 shows the curvilinear positions of the vehicles under the above supervisor; the gray area corresponds to the $[89,111] \mathrm{m}$ region. Note that vehicle 3 , which does not conflict with any other vehicle, crosses at its target speed. The accompanying video submission ${ }^{1}$ shows this example, as well as a larger instance of 25 vehicles to illustrate a more complex situation.

\section{B. Computation time}

The results from theorem 3 allows to use a relatively small time horizon in the simulator, which enables a fast iterative resolution of the FH-MPC formulation, even with a high number of vehicles. Figure 3 shows the distribution of the

\footnotetext{
${ }^{1}$ Also available at https://youtu.be/GF5KKr2V7.Sg
} 


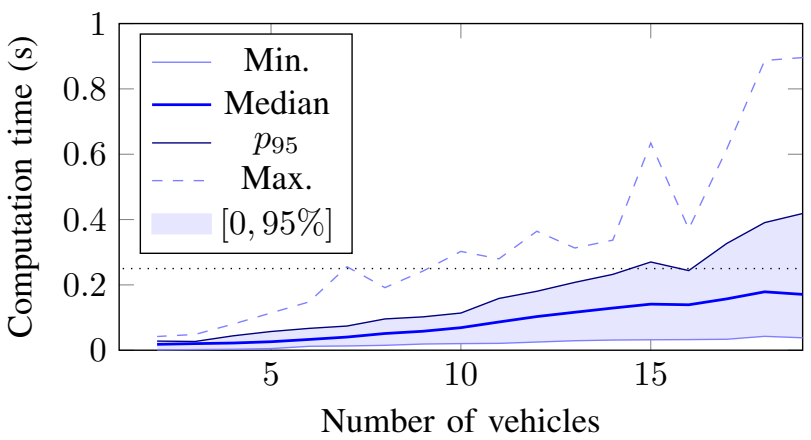

Fig. 3. Distribution of computation times for a given number of vehicles, for a time step duration of $0.25 \mathrm{~s}$.

computation time needed to solve the FH-MPC formulation for a given number of vehicles, over 10 different instances, for a time step duration of $0.25 \mathrm{~s}$. For up to 16 vehicles, this computation time is lower than the duration of a time step in $95 \%$ of the situations, which could allow near real-time applications.

\section{CONCLUSION}

In this article, we have proposed a novel approach to design a least restrictive and minimally deviating supervisor for the safe navigation of semi-autonomous vehicles through an intersection. The supervisor is tasked with overriding the desired controls of individual vehicles, only when necessary. Moreover, the overridden control of all vehicles should be as close as possible, for a given metric, to the controls initially requested. We proposed an infinite horizon model predictive control scheme to solve this optimal supervision problem. We then derived a finite horizon MPC formulation and, for a long enough horizon, we theoretically proved that the finite horizon MPC is ensured to maintain safety and avoid deadlocks for all future times. Finally, we implemented the supervisor using the commercial mixed-integer quadratic programming solver Gurobi jointly with the open-source traffic simulation tool SUMO to demonstrate these properties on a small set of 6 vehicles as well as on a larger instance comprising 25 vehicles.

Contrary to previous work on semi-autonomous supervision, our approach is based on the simultaneous resolution of the discrete problem of finding an optimal vehicle ordering, and the continuous problem of optimizing trajectories within this ordering. This method, using the concept of pairwise priorities [13] between vehicles, allows to consider more complex intersection geometries while taking into account second-order kinodynamics constraints, with potentially several vehicles behind one another or having merging or diverging paths. Since the supervisor only needs to consider a relatively short time horizon, it is able to run in real-time with at least a dozen vehicles.

This new approach to semi-autonomous intersection supervision opens up several possibilities for future research. Careful study of the hypotheses of the demonstrated theorems and the solutions of the optimization problem can help determine the best instant at which priorities should be assigned to vehicles. Using additional assumptions on vehicles behavior, some of the hypotheses can likely be relaxed to further improve the throughput of the intersection; different objective functions can be used to find solutions balancing respect of individual vehicles wishes with traffic efficiency for the intersection. Moreover, our formulation may be distributed among vehicles, which could provide a fast and efficient method for safe and fully autonomous intersection crossing.

\section{REFERENCES}

[1] A. Vahidi and A. Eskandarian, "Research advances in intelligent collision avoidance and adaptive cruise control," IEEE Transactions on Intelligent Transportation Systems, vol. 4, no. 3, pp. 143-153, sep 2003.

[2] E. Coelingh, A. Eidehall, and M. Bengtsson, "Collision Warning with Full Auto Brake and Pedestrian Detection - a practical example of Automatic Emergency Braking," in 13th International IEEE Conference on Intelligent Transportation Systems. IEEE, sep 2010, pp. 155-160.

[3] K. Dresner and P. Stone, "Multiagent traffic management: a reservation-based intersection control mechanism," in Proceedings of the Third International Joint Conference on Autonomous Agents and Multiagent Systems, 2004. IEEE Computer Society, July 2004, pp. 530-537.

[4] A. de La Fortelle, "Analysis of reservation algorithms for cooperative planning at intersections," in Proceedings of the 13th International IEEE Annual Conference on Intelligent Transportation Systems, I. ITSS, Ed., September 2010.

[5] X. Qian, J. Gregoire, F. Moutarde, and A. de La Fortelle, "Prioritybased coordination of autonomous and legacy vehicles at intersection,' Oct. 2014.

[6] T. Fraichard and H. Asama, "Inevitable Collision States. A Step Towards Safer Robots?" Proceedings 2003 IEEE/RSJ International Conference on Intelligent Robots and Systems (IROS 2003), vol. 1, no. October, pp. 388-393, 2003.

[7] A. Colombo and D. Del Vecchio, "Least Restrictive Supervisors for Intersection Collision Avoidance: A Scheduling Approach," IEEE Transactions on Automatic Control, vol. 60, no. 6, pp. 1515-1527, jun 2014.

[8] S. A. Reveliotis and E. Roszkowska, "On the Complexity of Maximally Permissive Deadlock Avoidance in Multi-Vehicle Traffic Systems," IEEE Transactions on Automatic Control, vol. 55, no. 7, pp. 1646-1651, jul 2010.

[9] L. Bruni, A. Colombo, and D. Del Vecchio, "Robust multi-agent collision avoidance through scheduling," in 52nd IEEE Conference on Decision and Control. IEEE, dec 2013, pp. 3944-3950.

[10] H. Ahn and D. Del Vecchio, "Semi-autonomous Intersection Collision Avoidance through Job-shop Scheduling," 2015. [Online]. Available: http://arxiv.org/abs/1510.07026

[11] G. R. de Campos, F. Della Rossa, and A. Colombo, "Optimal and least restrictive supervisory control: Safety verification methods for humandriven vehicles at traffic intersections," in 2015 54th IEEE Conference on Decision and Control (CDC). IEEE, dec 2015, pp. 1707-1712.

[12] J. Peng, "Coordinating Multiple Robots with Kinodynamic Constraints Along Specified Paths," The International Journal of Robotics Research, vol. 24, no. 4, pp. 295-310, apr 2005.

[13] J. Gregoire, "Priority-based coordination of mobile robots," Ph.D. dissertation, MINES ParisTech, 2014. [Online]. Available: http: //arxiv.org/abs/1410.0879

[14] F. Altché, X. Qian, and A. de La Fortelle, "Time-optimal Coordination of Mobile Robots along Specified Paths," arXiv preprint, vol. arXiv:1603.04610, mar 2016, submitted to IEEE IROS 2016. [Online]. Available: http://arxiv.org/abs/1603.04610

[15] D. Younger, "Minimum feedback arc sets for a directed graph," IEEE Transactions on Circuit Theory, vol. 10, no. 2, pp. 238-245, Jun 1963.

[16] Gurobi Optimization, Inc., "Gurobi optimizer reference manual," 2015. [Online]. Available: http://www.gurobi.com

[17] D. Krajzewicz, J. Erdmann, M. Behrisch, and L. Bieker, "Recent development and applications of SUMO - Simulation of Urban MObility," International Journal On Advances in Systems and Measurements, vol. 5, no. 3\&4, pp. 128-138, December 2012. 